\title{
Consistent collective decisions under majorities based on difference of votes
}

\author{
Mostapha Diss • \\ Patrizia Pérez-Asurmendi
}

\begin{abstract}
The main criticism to the aggregation of individual preferences under majority rules refers to the possibility of reaching inconsistent collective decisions from the election process. In these cases, the collective preference includes cycles and even could prevent the election of any alternative as the collective choice. The likelihood of consistent outcomes under a class of majority rules constitutes the aim of this paper. Specifically, we focus on majority rules that require certain consensus in individual preferences to declare an alternative as the winner. Under majorities based on difference of votes, the requirement asks to the winner alternative to obtain a difference in votes with respect to the loser alternative taking into account that individuals are endowed with weak preference orderings. Same requirement is asked to the restriction of these rules to individual linear preferences.
\end{abstract}

Keywords Majorities based on difference of votes · Probability · Transitivity · Triple-acyclicity.

JEL Classification: D70, D71

\section{Introduction}

Since Condorcet (1785) introduced the Voting Paradox, it is well known that the aggregation of transitive individual preferences under simple majority rule

\footnotetext{
M. Diss

Université de Lyon, Lyon, F-69007, France; CNRS, GATE Lyon Saint-Etienne, Ecully, F69130, France; Université Jean Monnet, Saint-Etienne, F-42000, France.

E-mail: diss@gate.cnrs.fr

Corresponding author: P. Pérez-Asurmendi

Grupo de investigación PRESAD, Universidad de Valladolid, Avda. Valle de Esgueva 6 , 47011, Valladolid, Spain

$\&$

Grupo de investigación SEED, Universidad Pública de Navarra, Campus de Arrosadia, 31006, Pamplona, Spain.

E-mail: patrizia.perez@eco.uva.es
} 
could lead to inconsistent collective preferences. Recalling the classical example, consider a three-alternative election with alternatives $x_{1}, x_{2}, x_{3}$ and three individuals endowed with the following rankings $x_{1} x_{2} x_{3}, x_{2} x_{3} x_{1}$ and $x_{3} x_{1} x_{2}$, where, for instance, $x_{1} x_{2} x_{3}$ means that $x_{1}$ is preferred to $x_{2}, x_{2}$ is preferred to $x_{3}$ and $x_{1}$ is preferred to $x_{3}$. For each pair of alternatives, each individual casts a vote for her/his preferred alternative following just assumed orderings. Adding up these votes, alternatives $x_{1}, x_{2}$ and $x_{3}$ defeat $x_{2}, x_{3}$ and $x_{1}$ respectively, by two votes to one. In that voting situation, there is a cycle on the ordering induced by the strict collective preference. In such a case, that preference fails on transitivity and on triple-acyclicity given the requirements of such conditions. To illustrate, assume that alternative $x_{1}$ defeats $x_{2}$ and $x_{2}$ defeats $x_{3} ; x_{1}$ defeats $x_{3}$ whenever the strict collective preference is transitive whereas $x_{3}$ does not defeat $x_{1}$ whenever the strict collective preference is triple-acyclic.

Consider now the following voting process' outcome: $x_{1}$ defeats $x_{2}$ and it is indifferent to $x_{3}$, and $x_{2}$ is also indifferent to $x_{3}$. In this case, the weak collective preference fails on consistency. Notice that the strict preference associated with that weak preference behaves right but the indifference relation associated with the weak preference fails on transitivity.

The idea that the Voting Paradox 'should rarely be observed in any real three-candidate elections with large electorates' stated by Gehrlein (2009), promotes the probabilistic study of the occurrence of that paradox and of their consequences under different aggregation rules. In several studies, it is assumed an a priori probability model to estimate the likelihood of different voting situations, derived the conditions under which the paradox or the effects of that appear and reached probabilities through combinatoric calculus. In this context, stand out the studies about simple majority rule (Gehrlein and Fishburn 1976; Fishburn and Gehrlein 1980; Gehrlein 1983), supermajority rules (Balasko and Crès 1997; Tovey 1997) or scoring rules (Gehrlein and Fishburn 1980, 1981, 1983; Cervone et al. 2005), among others.

This paper is devoted to analyze and compare the probabilities of consistent collective decisions over three alternatives for a class of majorities rules: majorities based on difference of votes (García-Lapresta and Llamazares 2001; Llamazares 2006; Houy 2007). Given two alternatives, these majorities based on differences focus on requiring to an alternative, to be declared the winner, to reach a number of votes that exceeds the number of votes for the other alternative in a quantity fixed before the voting process. In such majorities, individual preferences are understood as crisp preferences, i.e. given a pair of alternatives individuals declare if they prefer an alternative to another one or if they are indifferent between them. Here, we distinguish between the case where individuals are endowed with weak preferences and the case where individuals are endowed with linear orderings.

Coming back to the consistent collective decisions analyzed here, we specifically calculate the probabilities of transitive and triple-acyclic strict collective preferences and the corresponding ones of transitive weak collective preferences for the majorities based on difference of votes, as the proportion of collective 
voting situations giving rise to consistent collective decisions over the total number of possible voting situations.

To calculate the probabilities of consistent outcomes under majorities based on difference of votes taking into account weak and linear individual preferences respectively, we consider the Impartial Anonymous Culture (IAC) condition (Gehrlein and Fishburn 1976) to describe the likelihood of the possible individual orderings. On the one hand, assuming weak or linear individual orderings jointly with the IAC condition allow to know the total number of possible collective preferences (again, Gehrlein and Fishburn 1976). On the other hand, the number of consistent profiles is calculated by means of Ehrhart polynomials, a method recently introduced in the social choice literature by Wilson and Pritchard (2007) and Lepelley et al. (2008) in order to estimate the probabilities of some voting paradoxes under the IAC condition.

The methodology proposed here allows us to find the needed thresholds which guarantee that the probability of consistent outcomes is close to 1 . In addition, we are able to hypothesize about the relationship between the type of individual preferences assumed and the likelihood of consistent collective decisions. Moreover, we set forth our results for majorities based on difference of votes with previous ones on simple majority rule (Gehrlein (1997) and Lepelley and Martin (2001)) and supermajority rules (Ferejohn and Grether (1974)).

The paper is organized as follows. Section 2 describes the theoretical framework followed in this paper and introduces majorities based on difference of votes. Sections 3 and 4 provide the results about the probability of consistent collective decisions under majorities based on difference of votes with linear preferences and with weak preferences, respectively. Section 5 concludes.

\section{Preliminaries}

Consider a set of three alternatives $X=\left\{x_{1}, x_{2}, x_{3}\right\}$ in an election with $m$ individuals. Let $S$ be a binary relation on $X$, i.e. a subset of the cartesian product $X \times X$. In what follows, $x_{i} S x_{j}$ stands for $\left(x_{i}, x_{j}\right) \in S$, i.e. when $x_{i}$ is in the relation $S$ with $x_{j} . S^{-1}$ is the inverse relation of $S$ defined by $x_{i} S^{-1} x_{j} \Leftrightarrow$ $x_{j} S x_{j}$ and $S^{c}$ is the complement relation of $S$ defined by $x_{i} S^{c} x_{j} \Leftrightarrow \neg\left(x_{i} S x_{j}\right)$. Given two binary relations $S$ and $T$, the intersection of $S$ and $T$ is also a binary relation defined by $x_{i}(S \cap T) x_{j} \Leftrightarrow\left(x_{i} S x_{j} \wedge x_{i} T x_{j}\right)$. A binary relation $S$ on $X$ is

1. reflexive if $\forall x \in X, x S x$,

2. symmetric if $\forall x_{i}, x_{j} \in X, x_{i} S x_{j} \Rightarrow x_{j} S x_{i}$,

3. asymmetric if $\forall x_{i}, x_{j} \in X, x_{i} S x_{j} \Rightarrow \neg\left(x_{j} S x_{i}\right)$,

4. antisymmetric if $\forall x_{i}, x_{j} \in X,\left(x_{i} S x_{j} \wedge x_{j} S x_{i}\right) \Rightarrow x_{i}=x_{j}$,

5. complete if $\forall x_{i}, x_{j} \in X, x_{i} S x_{j} \vee x_{j} S x_{i}$,

6. transitive if $\forall x_{i}, x_{j}, x_{l} \in X,\left(x_{i} S x_{j} \wedge x_{j} S x_{l}\right) \Rightarrow x_{i} S x_{l}$,

7. triple-acyclic if $\forall x_{i}, x_{j}, x_{l} \in X,\left(x_{i} S x_{j} \wedge x_{j} S x_{l}\right) \Rightarrow \neg\left(x_{l} S x_{i}\right)$.

A weak preference $R$ is a complete binary relation on the set of alternatives

$X$. The strict preference $P$ associated with $R$ is the asymmetric binary relation 
on $X$ defined by $P=\left(R^{-1}\right)^{c}$ and the corresponding indifference relation $I$ is the reflexive and symmetric binary relation on $X$ defined by $I=R \cap R^{-1}$. $\mathcal{P}(X)$ is the set of strict preferences. A weak ordering is a transitive weak preference whereas a linear ordering is also antisymmetric.

From definitions above it is well know that any weak ordering implies a transitive strict preference relation and a transitive indifference relation. Moreover, any transitive strict preference is also a triple-acyclic preference relation. Notice that the converse is not true.

Given that the social decision between two alternatives is given by either a strict preference relation or an indifference relation, and that three alternatives are in contest, we consider the 27 cases in Table 1 as possible social outcomes.

Table 1 Possible social outcomes in a three-alternative election.

\begin{tabular}{|c|c|c|c|c|c|c|c|}
\hline 1. & $x_{1} P x_{2}$ & $x_{2} P x_{3}$ & $x_{1} P x_{3}$ & 14 & $x_{1} P x_{2}$ & $x_{2} I x_{3}$ & $x_{1} I x_{3}$ \\
\hline 2. & $x_{1} P x_{3}$ & $x_{3} P x_{2}$ & $x_{1} P x_{2}$ & 15 . & $x_{1} I x_{2}$ & $x_{2} I x_{3}$ & $x_{1} P x_{3}$ \\
\hline 3. & $x_{2} P x_{1}$ & $x_{1} P x_{3}$ & $x_{2} P x_{3}$ & 16 & $x_{1} I x_{2}$ & $x_{2} I x_{3}$ & $x_{3} P x_{1}$ \\
\hline 4. & $x_{2} P x_{3}$ & $x_{3} P x_{1}$ & $x_{2} P x_{1}$ & 17 & $x_{1} I x_{3}$ & $x_{3} P x_{2}$ & $x_{1} I x_{2}$ \\
\hline 5. & $x_{3} P x_{1}$ & $x_{1} P x_{2}$ & $x_{3} P x_{2}$ & 18 & $x_{2} P x_{1}$ & $x_{1} I x_{3}$ & $x_{2} I x_{3}$ \\
\hline 6. & $x_{3} P x_{2}$ & $x_{2} P x_{1}$ & $x_{3} P x_{1}$ & 19 & $x_{1} I x_{2}$ & $x_{2} P x_{3}$ & $x_{1} I x_{3}$ \\
\hline & $x_{1} P x_{2}$ & $x_{2} I x_{3}$ & $x_{1} P x_{3}$ & 20 & $x_{1} P x_{2}$ & $x_{2} P x_{3}$ & $x_{1} I x_{3}$ \\
\hline & $x_{2} P x_{1}$ & $x_{1} I x_{3}$ & $x_{2} P x_{3}$ & 21 & $x_{3} P x_{1}$ & $x_{1} P x_{2}$ & $x_{2} I x_{3}$ \\
\hline & $x_{3} P x_{1}$ & $x_{1} I x_{2}$ & $x_{3} P x_{2}$ & 22 & $x_{2} P x_{3}$ & $x_{3} P x_{1}$ & $x_{1} I x_{2}$ \\
\hline 10. & $x_{1} I x_{2}$ & $x_{2} P x_{3}$ & $x_{1} P x_{3}$ & 23 & $x_{3} P x_{2}$ & $x_{1} P x_{3}$ & $x_{1} I x_{2}$ \\
\hline & $x_{2} I x_{3}$ & $x_{3} P x_{1}$ & $x_{2} P x_{1}$ & 24 & $x_{2} P x_{1}$ & $x_{1} P x_{3}$ & $x_{2} I x_{3}$ \\
\hline 12 . & $x_{1} I x_{3}$ & $x_{3} P x_{2}$ & $x_{1} P x_{2}$ & 25 & $x_{3} P x_{2}$ & $x_{2} P x_{1}$ & $x_{1} I x_{3}$ \\
\hline \multirow{2}{*}{\multicolumn{2}{|c|}{13.}} & $x_{2} I x_{3}$ & $x_{1} I x_{3}$ & 26 & $x_{1} P x_{2}$ & $x_{2} P x_{3}$ & $x_{3} P x_{1}$ \\
\hline & & & & 27. & $x_{2} P x_{1}$ & $x_{1} P x_{3}$ & $x_{3} P x_{2}$ \\
\hline
\end{tabular}

Our interest focuses on the frequency of consistent social outcomes given the 27 possible outcomes above. We distinguish among three cases of consistent outcomes; the case of weak orderings corresponding to the first thirteen outcomes, the case of transitive strict preferences corresponding to the first nineteen and the case of triple-acyclic strict preferences corresponding to the first twenty-five outcomes.

\subsection{Individual preferences}

We consider that individuals compare the alternatives on $X$ by pairs and declare their preferences by means of values $r_{i j}^{p} \in\{0,0.5,1\}$. That is, individuals show their preferences through crisp preferences. In such a case, if $r_{i j}^{p}=1$, individual $p$ prefers alternative $x_{i}$ to alternative $x_{j}$, whereas if $r_{i j}^{p}=0$, individual $p$ prefers $x_{j}$ to $x_{i}$. If $r_{i j}^{p}=0.5$, individual $p$ is indifferent between both alternatives. Condition $r_{i j}^{p}+r_{j i}^{p}=1$ guarantees that the preference of 
individual $p$ is a weak preference. Moreover, the conditions

$$
\begin{aligned}
& \left(r_{i j}^{p}=1 \wedge r_{j l}^{p}=1\right) \Rightarrow r_{i l}^{p}=1, \\
& \left(r_{i j}^{p}=0.5 \wedge r_{j l}^{p}=0.5\right) \Rightarrow r_{i l}^{p}=0.5,
\end{aligned}
$$

assure that the preference of individual $p$ is a weak ordering. Individual linear orderings could also be represented in this framework by including the following condition: $r_{i j}^{p} \in\{0,1\} \forall i \neq j$. Thus, individuals could only be indifferent between an alternative and itself.

The preferences of each individual over the alternatives in $X=\left\{x_{1}, x_{2}, x_{3}\right\}$ can be represented using a $3 \times 3$ matrix $R^{p}=\left(r_{i j}^{p}\right)$ as follows:

$$
R^{p}=\left(\begin{array}{ccc}
0.5 & r_{12}^{p} & r_{13}^{p} \\
1-r_{12}^{p} & 0.5 & r_{23}^{p} \\
1-r_{13}^{p} & 1-r_{23}^{p} & 0.5
\end{array}\right)
$$

Individual preferences are collected in a vector where each vector-element represents the preferences of an individual. Assuming $m$ individuals ${ }^{1}$ and taking into account the above distinction among linear and weak preferences, a profile of linear orderings is a vector $\left(R^{1}, \ldots, R^{m}\right) \in \mathcal{L}(X)^{m}$, being $\mathcal{L}(X)$ the set of all linear orderings; and a profile of weak orderings is a vector $\left(R^{1}, \ldots, R^{m}\right) \in \mathcal{W}(X)^{m}$, being $\mathcal{W}(X)$ the set of all weak orderings.

\subsection{Majorities based on difference of votes}

The concept and the definition of majorities based on difference of votes was introduced in García-Lapresta and Llamazares (2001) and was later axiomatically characterized in Llamazares (2006), and subsequently in Houy (2007). These rules involve crisp preferences, i.e. given a pair of alternatives, individuals could declare their preference for one of them or their indifference between both alternatives.

Under these majorities, an alternative, say $x_{i}$, is declared the winner if the number of individuals who prefer that alternative, to the other one, say $x_{j}$, exceeds the number of individuals who prefer $x_{j}$ to $x_{i}$ in a difference of votes, fixed before the election process. Assuming $m$ individuals, that difference could take any integer value in $\{0, \ldots, m-1\}$.

In what follows, the symbol \# stands for the cardinality of a set.

\section{Definition 1 (Majorities based on difference of votes)}

Given $k \in\{0,1, \ldots, m-1\}$, the majority based on difference of votes or $M_{k}$ majority is the mapping $M_{k}: \mathcal{W}(X)^{m} \longrightarrow \mathcal{P}(X)$ defined by $M_{k}\left(R^{1}, \ldots, R^{m}\right)=P_{k}$, where

$$
x_{i} P_{k} x_{j} \Leftrightarrow \#\left\{p \mid r_{i j}^{p}=1\right\}>\#\left\{p \mid r_{j i}^{p}=1\right\}+k .
$$

1 To calculate the probabilities presented here, $m$ takes the following values: $3,4,5,10$, $100,1,000$ and 100,000. 
The indifference relation associated with $P_{k}$ is as follows:

$$
x_{i} I_{k} x_{j} \Leftrightarrow\left|\#\left\{p \mid r_{i j}^{p}=1\right\}-\#\left\{p \mid r_{j i}^{p}=1\right\}\right| \leq k .
$$

Example 1 Let $R^{I}$ and $R^{I I}$ be the following individual weak preference orderings over the alternatives on $X=\left\{x_{1}, x_{2}, x_{3}\right\}$.

$$
R^{I}=\left(\begin{array}{ccc}
0.5 & 1 & 0.5 \\
0 & 0.5 & 0 \\
0.5 & 1 & 0.5
\end{array}\right), \quad R^{I I}=\left(\begin{array}{ccc}
0.5 & 0.5 & 1 \\
0.5 & 0.5 & 1 \\
0 & 0 & 0.5
\end{array}\right)
$$

Consider the profile $\left(R^{1}, R^{2}, R^{3}, R^{4}, R^{5}\right)$ where

$$
R^{p}= \begin{cases}R^{I} & \text { if } p=1,2,3, \\ R^{I I} & \text { if } p=4,5\end{cases}
$$

Assuming a required difference of votes $k$ equal to 2 and applying the corresponding $M_{2}$ majority, we have

$$
\begin{gathered}
\#\left\{p \mid r_{12}^{p}=1\right\}=3>\#\left\{p \mid r_{21}^{p}=1\right\}+2=0+2 \Rightarrow x_{1} P_{2} x_{2}, \\
\left|\#\left\{p \mid r_{23}^{p}=1\right\}-\#\left\{p \mid r_{32}^{p}=1\right\}\right|=|2-3| \leq 2 \Rightarrow x_{2} I_{2} x_{3} \\
\left|\#\left\{p \mid r_{13}^{p}=1\right\}-\#\left\{p \mid r_{31}^{p}=1\right\}\right|=|2-0| \leq 2 \Rightarrow x_{1} I_{2} x_{3}
\end{gathered}
$$

Majorities based on difference of votes are located between simple majority rule where the difference of votes is zero and unanimity where the difference of votes is the total number of individuals $m$ minus one. Moreover, if the indifference state is ruled out from individual preferences, these majorities are equivalent to supermajority rules. ${ }^{2}$ In this regard, notice that if $r_{i j}^{p} \in\{0,1\}$, then $\#\left\{p \mid r_{j i}^{p}=1\right\}=\#\left\{p \mid r_{i j}^{p}=0\right\}=m-\#\left\{p \mid r_{i j}^{p}=1\right\}$. Therefore, under majorities based on difference of votes we have

$$
\begin{aligned}
\#\left\{p \mid r_{i j}^{p}=1\right\}>m-\#\left\{p \mid r_{i j}^{p}=1\right\}+k & \Leftrightarrow 2 \#\left\{p \mid r_{i j}^{p}=1\right\}>m+k \\
& \Leftrightarrow \#\left\{p \mid r_{i j}^{p}=1\right\}>\frac{m+k}{2} \\
& \Leftrightarrow \frac{\#\left\{p \mid r_{i j}^{p}=1\right\}}{m}>\frac{m+k}{2 m} \\
& \Leftrightarrow \frac{\#\left\{p \mid r_{i j}^{p}=1\right\}}{m}>0.5+\frac{k}{2 m}
\end{aligned}
$$

which is analogous to the result under supermajority rules.

In what follows, the restriction of $M_{k}$ majorities to $\mathcal{L}(X)^{m}$, i.e. $\left.M_{k}\right|_{\mathcal{L}(X)^{m}}$, is referred to as $M_{k}^{L}$ majorities.

2 Given $\tau>0.5$, supermajority rules are defined by $x_{i} P^{\tau} x_{j} \Leftrightarrow \frac{\#\left\{p \mid r_{i j}^{p}=1\right\}}{m} \geq \tau$. 
Example 2 Let $R^{I}$ and $R^{I I}$ be the following individual linear preference orderings over the alternatives on $X=\left\{x_{1}, x_{2}, x_{3}\right\}$.

$$
R^{I}=\left(\begin{array}{ccc}
0.5 & 1 & 1 \\
0 & 0.5 & 1 \\
0 & 0 & 0.5
\end{array}\right), \quad R^{I I}=\left(\begin{array}{ccc}
0.5 & 0 & 1 \\
1 & 0.5 & 1 \\
0 & 0 & 0.5
\end{array}\right)
$$

Consider the profile $\left(R^{1}, R^{2}, R^{3}, R^{4}, R^{5}\right)$ where

$$
R^{p}= \begin{cases}R^{I} & \text { if } p=1,2,3 \\ R^{I I} & \text { if } p=4,5\end{cases}
$$

Assuming a required difference of votes $k$ equal to 1 and applying the corresponding $M_{1}^{L}$ majority, we have

$$
\begin{aligned}
& \left|\#\left\{p \mid r_{12}^{p}=1\right\}-\#\left\{p \mid r_{21}^{p}=1\right\}\right|=|3-2| \leq 1 \Rightarrow x_{1} I_{1}^{L} x_{2}, \\
& \#\left\{p \mid r_{23}^{p}=1\right\}=5>\#\left\{p \mid r_{32}^{p}=1\right\}+1=0+1 \Rightarrow x_{2} P_{1}^{L} x_{3} \\
& \#\left\{p \mid r_{13}^{p}=1\right\}=5>\#\left\{p \mid r_{31}^{p}=1\right\}+1=0+1 \Rightarrow x_{1} P_{1}^{L} x_{3}
\end{aligned}
$$

\section{Probability of consistent collective decisions under majorities based on difference of votes with linear orderings}

In this section the results about the probabilities of consistent collective decisions under $M_{k}^{L}$ majorities are introduced under IAC assumption. Given that voters are endowed with complete linear preference orderings, there are six possible preference orders that they might have,

$$
\begin{array}{lll}
x_{1} x_{2} x_{3}\left(m_{1}\right) & x_{1} x_{3} x_{2}\left(m_{2}\right) & x_{2} x_{1} x_{3}\left(m_{3}\right) \\
x_{2} x_{3} x_{1}\left(m_{4}\right) & x_{3} x_{1} x_{2}\left(m_{5}\right) & x_{3} x_{2} x_{1}\left(m_{6}\right)
\end{array}
$$

where $m_{i}$ is the number of voters with the associated linear preference ordering. In this framework, a voting situation is a vector $\boldsymbol{m}=\left(m_{1}, m_{2}, m_{3}, m_{4}, m_{5}, m_{6}\right)$ such that $\sum_{i=1}^{6} m_{i}=m$. As the IAC condition is assumed, all possible voting situations $\boldsymbol{m}$ are equally liked to be observed. Gehrlein and Fishburn (1976) showed that for $m$ agents and 3 alternatives, the total number of voting situations $\boldsymbol{m}$ is given by the expression:

$$
\psi(m)=\frac{(m+1)(m+2)(m+3)(m+4)(m+5)}{120} .
$$


3.1 Probabilities of triple-acyclic strict preferences under majorities based on difference of votes with linear orderings

To calculate the probability of triple-acyclic strict preferences under $M_{k}^{L}$ majorities, we focus on the cases from 1 to 25 in Table 1 . Specifically, we first calculate the probability of cyclic strict preferences, i.e. the probability of having preferences like the ones described in the cases 26 and 27 (see again Table 1). Thereafter, we obtain the probability of triple-acyclic cases as 1 minus the probability of cyclic strict preferences.

Going deeper on the strict preference described in the case 26, we notice that for such preference to exist, the numbers of voters associated with the linear orderings described in (2) have to fulfil the following conditions: $m_{1}+$ $m_{2}-m_{3}-m_{4}+m_{5}-m_{6}>k, m_{1}-m_{2}+m_{3}+m_{4}-m_{5}-m_{6}>k$ and $-m_{1}-m_{2}-m_{3}+m_{4}+m_{5}+m_{6}>k$.

In other words, the strict preference in the case 26 requires a voting situation $\boldsymbol{m}=\left(m_{1}, m_{2}, m_{3}, m_{4}, m_{5}, m_{6}\right)$ that fulfils the conditions given by the system of inequalities below.

$$
\left(x_{1} P_{k}^{L} x_{2}, x_{2} P_{k}^{L} x_{3} \text { and } x_{3} P_{k}^{L} x_{1}\right) \Rightarrow\left\{\begin{array}{l}
m_{1}+m_{2}-m_{3}-m_{4}+m_{5}-m_{6}>k, \\
m_{1}-m_{2}+m_{3}+m_{4}-m_{5}-m_{6}>k, \\
-m_{1}-m_{2}-m_{3}+m_{4}+m_{5}+m_{6}>k, \\
m_{i} \geq 0 \text { for } i \in\{1, \ldots, 6\} \\
m-1 \geq k \geq 0, \\
m_{1}+m_{2}+m_{3}+m_{4}+m_{5}+m_{6}=m .
\end{array}\right.
$$

Therefore, to calculate the probability of cyclic strict preferences, we need to solve the system of linear inequalities derived from conditions that the numbers of voters associated with the linear orderings in (2) have to hold for strict preferences like the ones in the cases 26 and 27 to exist.

We compute the number of voting situations that fulfil these conditions by means of the Parameterized Barvinok's algorithm (Verdoolaege et al. (2004)) ${ }^{3}$. Such algorithm allows to quantify the number of integer solutions for systems of inequalities with parameters. The connection of such algorithm to Social Choice Theory was recently pointed out by Wilson and Pritchard (2007) and Lepelley et al. (2008).

Given the two parameters $m$ and $k$, the number of voting situations $\boldsymbol{m}$ for our system is given by bivariate quasi polynomials in $m$ and $k$ with 2 periodic coefficients meaning that such coefficients depend on the parity of the parameters $m$ and $k$. Following the notation introduced in Lepelley et al. (2008), we represent these coefficients by a list of 2 rational numbers enclosed in square brackets. To illustrate, assume the bracketed list $\left[[a, b]_{m},[b, a]_{m}\right]_{k}$. In the case of even $k$, the relevant list corresponds to $[a, b]_{m}$. The coefficient will be either $a$ when $m$ is even or $b$ when $m$ is odd. Accordingly, in the case

3 The free software to calculate the integer points under the Parameterized Barvinok's algorithm can be found in http://freecode.com/projects/barvinok. 
of odd $k$, the relevant list is $[b, a]_{m}$ and therefore, the coefficient will be either $b$ when $m$ is even or $a$ when $m$ is odd. Thus, the coefficient will be $a$ when $m$ and $k$ have the same parity and $b$ otherwise.

Notice that in the case of the cyclical strict preferences depicted in the cases 26 and 27 (Table 1), the number of solutions in the system of inequalities derived from the strict preference in the case 26 is the same as in the system derived from the strict preference in the case 27 given the symmetry of such cases. The program indicates that the corresponding quasi polynomial for each of these cases is as follows:

$$
-\frac{81}{1280} k^{5}+Q_{1}(m, k) k^{4}+Q_{2}(m, k) k^{3}+Q_{3}(m, k) k^{2}+Q_{4}(m, k) k+Q_{5}(m, k),
$$

where

$$
\begin{aligned}
& Q_{1}(m, k)=\frac{27}{256} m+\left[\left[0, \frac{81}{256}\right]_{m},\left[\frac{81}{256}, 0\right]_{m}\right]_{k} \\
& Q_{2}(m, k)=-\frac{9}{128} m^{2}+\left[\left[0,-\frac{27}{64}\right]_{m},\left[-\frac{27}{64}, 0\right]_{m}\right]_{k} m \\
& +\left[\left[\frac{9}{64},-\frac{63}{128}\right]_{m},\left[-\frac{63}{128}, \frac{9}{64}\right]_{m}\right]_{k} \\
& Q_{3}(m, k)=\frac{3}{128} m^{3}+\left[\left[0, \frac{27}{128}\right]_{m},\left[\frac{27}{128}, 0\right]_{m}\right]_{k} m^{2} \\
& +\left[\left[-\frac{9}{64}, \frac{63}{128}\right]_{m},\left[\frac{63}{128},-\frac{9}{64}\right]_{m}\right]_{k} m \\
& +\left[\left[0, \frac{27}{128}\right]_{m},\left[\frac{27}{128}, 0\right]_{m}\right]_{k} \\
& Q_{4}(m, k)=-\frac{1}{256} m^{4}+\left[\left[0,-\frac{3}{64}\right]_{m},\left[-\frac{3}{64}, 0\right]_{m}\right]_{k} m^{3} \\
& +\left[\left[\frac{3}{64},-\frac{21}{128}\right]_{m},\left[-\frac{21}{128}, \frac{3}{64}\right]_{m}\right]_{k} m^{2} \\
& +\left[\left[0,-\frac{9}{64}\right]_{m},\left[-\frac{9}{64}, 0\right]_{m}\right]_{k} m \\
& +\left[\left[-\frac{1}{20}, \frac{71}{1280}\right]_{m},\left[\frac{71}{1280},-\frac{1}{20}\right]_{m}\right]_{k}
\end{aligned}
$$




$$
\begin{aligned}
Q_{5}(m, k)= & \frac{1}{3840} m^{5}+\left[\left[0, \frac{1}{256}\right]_{m},\left[\frac{1}{256}, 0\right]_{m}\right]_{k} m^{4} \\
& +\left[\left[-\frac{1}{192}, \frac{7}{384}\right]_{m},\left[\frac{7}{384},-\frac{1}{192}\right]_{m}\right]_{k} m^{3} \\
& +\left[\left[0, \frac{3}{128}\right]_{m},\left[\frac{3}{128}, 0\right]_{m}\right]_{k} m^{2} \\
& +\left[\left[\frac{1}{60},-\frac{71}{3840}\right]_{m},\left[-\frac{71}{3840}, \frac{1}{60}\right]_{m}\right]_{k} m \\
& +\left[\left[0,-\frac{7}{256}\right]_{m},\left[-\frac{7}{256}, 0\right]_{m}\right]_{k}
\end{aligned}
$$

In addition, the program points out that this relation holds only if $k \leq$ $(m-3) / 3$. Otherwise, the number of voting situations is zero.

We simplify ${ }^{4}$ the quasi polynomial above by considering different values of $m$ and $k$. Thus, it can be deduced that the number of voting situations corresponding to each of the strict preferences represented by the cases 26 and 27 in Table 1 is given by $F_{1}(m, k)$ if both $m$ and $k$ are odd (or even) and by $F_{2}(m, k)$ if one of the parameters $(m$ or $k)$ is odd and the other one is even such that:

$$
\begin{aligned}
F_{1}(m, k)= & \frac{1}{3840}((m-3 k-4)(m-3 k)(m-3 k+4) \\
& (m-3 k-2)(m-3 k+2)) . \\
F_{2}(m, k)= & \frac{1}{3840}((m-3 k+3)(m-3 k+7)(m-3 k+1) \\
& (m-3 k+5)(m-3 k-1)) .
\end{aligned}
$$

As a consequence of the above number of voting situations and taking into account the symmetry of the strict preferences of the cases 26 and 27 in Table 1 and the total number of voting situations $\psi(m)$ in $(3)$, we introduce the probabilities of having triple-acyclic strict preferences under $M_{k}^{L}$ majorities in the following result.

Proposition 1 Consider a three-candidate election with $m$ voters under $M_{k}^{L}$ majority rules where each individual vote consists of a linear preference ordering on the candidates. Assuming that all voting situations are equally likely $(I A C)$, if $k \leq(m-3) / 3$, the probability of triple-acyclic strict preference is as follows:

\footnotetext{
4 Such simplification is done with Maple software.
} 
- If both $m$ and $k$ are odd (or even):

$$
1-\frac{2 F_{1}(m, k)}{\psi(m)}
$$

- If one of the parameters ( $m$ or $k$ ) is odd and the other one is even:

$$
1-\frac{2 F_{2}(m, k)}{\psi(m)}
$$

Computed values of this probability are listed in Table 2. The values of the difference of votes $k$ correspond to the ones that provide a probability of triple-acyclic strict preferences equal to 1 . As it is previously mentioned, these probabilities indicate that the number of solutions in the systems of inequalities corresponding to the cyclic strict preferences (cases 26 and 27 in Table 1) are equal to zero when $k>(m-3) / 3$.

Table 2 Probability of triple-acyclic $P_{k}^{L}$.

\begin{tabular}{||r|rrrrrrr||}
\hline $\begin{array}{r}m \rightarrow \\
k \downarrow\end{array}$ & 3 & 4 & 5 & 10 & 100 & 1,000 & 100,000 \\
\hline 0 & 0.9643 & 1 & 0.9524 & 0.9860 & 0.9462 & 0.9384 & 0.9375 \\
1 & 1 & & 1 & 0.9860 & 0.9462 & 0.9384 & 0.9375 \\
2 & & & & 1 & 0.9605 & 0.9403 & 0.9375 \\
32 & & & & & 1 & 0.9628 & 0.9378 \\
332 & & & & & & 1 & 0.9406 \\
33,332 & & & & & & & 1 \\
\hline
\end{tabular}

Attending to the probabilities in Table 2, notice that, in the case of $k=0$, the probability $0.9375=15 / 16$, as well as 0.9643 and 0.9524 , are in accordance with the results previously obtained by Gehrlein (1997) when the number of voters tends to infinity and equals 3 and 5 , respectively.

As we noted before, $M_{k}^{L}$ majorities are equivalent to supermajorities. Ferejohn and Grether (1974) proved that supermajorities are acyclic whenever $1>\tau \geq(\# X-1) / \# X$. Therefore, in the three-alternative case, we have that $(\# X-1) / \# X=2 / 3$. Bearing in mind that $\tau=0,5+k / 2 m$, the probability of triple-acyclic $P_{k}^{L}$ equals 1 when $\tau=2 / 3$ for $m=3, m=100, m=1,000$, and $m=100,000$. In the cases of $m=4, m=5$, and $m=10$ we have that $\tau=0.6$. Hence, our probabilistic results do not contradict the above theoretical one.

Furthermore, some other interesting facts could be emphasized from the above probabilities.

First, $M_{0}^{L}$ majority provide a probability of triple-acyclic strict preferences equal to 1 for the case of $m=4$ whereas a difference of votes equal to 1 is necessary in the case of $m=3$ and $m=5$ to achieve such probability. We conjecture that this odd result attends to the fact that the likelihood of having ties is greater in the case of an even number of individuals than in the case 
of an odd number of individuals when these voters are endowed with linear orderings.

Second, the weight of the difference of votes necessary to achieve a probability of triple-acyclic strict preferences over the total number of votes equal to 1 , understood as the percentage of $k$ over $m$, increases with the number of individuals from $m=10$ to $m=100,000$. In the case of $m=10$ required difference signifies a $20 \%$ of the value of $m$, a $32 \%$ in the case of $m=100$, a $33.2 \%$ in the case of $m=1,000$ and a $33.332 \%$ in the case of $m=100,000$.

Third, the probabilities do not reflect small changes in the magnitude of the thresholds. See for instance, the probabilities attached to the cases $m=10$, $m=100$ and $m=1,000$ for the values of the difference $k$ equal to 0 and 1 and for the case of $m=100,000$ in the case of $k$ equal 0,1 and 2 .

Finally, triple-acyclic strict preferences under $M_{k}^{L}$ majorities can be guaranteed with a probability of 1 for not too demanding differences of votes.

Notice that it is possible to represent these probabilities as a function of the required difference of votes divided by the total number of votes, i.e., the relative threshold. Let $\alpha=k / m$ be such threshold. If we assume large electorates - that is, $m$ tends to infinity - and replace $k$ by $\alpha m$ in the results, the desired probabilities are obtained as a function of $\alpha$ that only considers the term of higher degree in $m$. Then, the following corollary is deduced from the results in Proposition 1.

Corollary 1 For large electorates $(m \rightarrow \infty)$, the probability of triple-acyclic strict preference is as follows:

1. If $\alpha<1 / 3$

$$
1-\frac{(1-3 \alpha)^{3}}{16}
$$

2. If $\alpha \geq 1 / 3$

1.

Notice again that we recover the result of Ferejohn and Grether (1974) about acyclicity since $\alpha \geq 1 / 3$ is equivalent to $1>\tau \geq 2 / 3$.

Table 3 Probability of triple-acyclic $P_{k}^{L}$ for large electorates.

\begin{tabular}{||r|rrrrrrrrrrrr||}
\hline$\alpha$ & 0 & 0.1 & 0.2 & 0.3 & $1 / 3$ & 0.4 & 0.5 & 0.6 & 0.7 & 0.8 & 0.9 & 0.99 \\
\hline & 0.9375 & 0.9895 & 0.9994 & 1.0000 & 1 & 1 & 1 & 1 & 1 & 1 & 1 & 1 \\
\hline
\end{tabular}

In Table 3, we present numerical values of the probability of triple-acyclic strict preferences for large electorates, as a function of the relative threshold $\alpha$. For $\alpha=0$, we obviously recover the well-known result that, under IAC, the probability that a cycle occurs in three-alternative elections is 1/16 (Gehrlein and Fishburn (1976)). These results illustrate that the probabilities increase as $\alpha$ rises. Moreover, the probabilities converge quickly to 1 as $\alpha$ tends to $1 / 3$. 
3.2 Probabilities of transitive strict preferences under majorities based on difference of votes with linear orderings

To study the probability of transitive strict preferences under $M_{k}^{L}$ majorities, we follow the same methodology as the one applied in Subsection 3.1 adding the cases 20,21,22,23, 24 and 25 in Table 1 to the outcomes 26 and 27 analyzed in the case of triple-acyclic strict preferences. Once we calculate the probability of non-transitive strict preferences collected in the cases from 20 to 27 , we determine the probability of transitive strict preferences as 1 minus the previous probability.

Ordinary preferences collected in cases from 20 to 25 are similar and hence, the number of integer solutions given by Barvinok's algorithm is the same in each of the six systems of inequalities representing these strict preferences.

For these cases, two validity domains can be distinguished. On the one hand, if $k \leq(m-3) / 3$, the number of voting situations is given by $G_{1}(m, k)$ if both $m$ and $k$ are odd (or even) and by $G_{2}(m, k)$ if one of the parameters ( $m$ or $k$ ) is odd and the other one is even such that:

$$
\begin{aligned}
G_{1}(m, k)= & \frac{1}{1920}(k+1)\left(121 k^{4}-116 k^{3}-200 m k^{3}+180 m k^{2}-\right. \\
& 164 k^{2}+130 m^{2} k^{2}+144 k-40 m^{3} k-100 m^{2} k+120 m k- \\
& \left.20 m^{2}-80 m+5 m^{4}+20 m^{3}\right) . \\
G_{2}(m, k)= & \frac{1}{1920} k\left(121 k^{4}-200 m k^{3}-600 k^{3}+130 m^{2} k^{2}+780 m k^{2}+\right. \\
& 910 k^{2}-40 m^{3} k-360 m^{2} k-840 m k-360 k+5 m^{4}+ \\
& \left.60 m^{3}+210 m^{2}+180 m-71\right) .
\end{aligned}
$$

On the other hand, if $(m-2) / 3 \leq k \leq m-2$, the number of voting situations is given by $G_{3}(m, k)$ if both $m$ and $k$ are odd (or even) and by $G_{4}(m, k)$ if one of the parameters $(m$ or $k$ ) is odd and the other one is even such that:

$$
\begin{aligned}
G_{3}(m, k)= & \frac{1}{3840}((m-k-2)(m-k+4)(m-k) \\
& (m-k+6)(m-k+2)) . \\
G_{4}(m, k)= & \frac{1}{3840}((m-k+7)(m-k+3)(m-k-1) \\
& (m-k+5)(m-k+1)) .
\end{aligned}
$$

Bearing in mind above numbers of voting situations, the results in Proposition 1 and the total number of voting situations $\psi(m)$ in $(3)$, we derive the probability of transitive strict preferences under $M_{k}^{L}$ majorities as follows. 
Proposition 2 Consider a three-candidate election with $m$ voters under $M_{k}^{L}$ majority rule where each individual vote consists of a linear preference ordering on the candidates. Assuming that all voting situations are equally likely (IAC), the probability of transitive strict preferences is as follows:

1. If $k \leq(m-3) / 3$

- If both $m$ and $k$ are odd (or even):

$$
1-\frac{2 F_{1}(m, k)+6 G_{1}(m, k)}{\psi(m)} .
$$

- If one of the parameters ( $m$ or $k$ ) is odd and the other is even:

$$
1-\frac{2 F_{2}(m, k)+6 G_{2}(m, k)}{\psi(m)} .
$$

2. If $(m-2) / 3 \leq k \leq m-2$

- If both $m$ and $k$ are odd (or even):

$$
1-\frac{6 G_{3}(m, k)}{\psi(m)} .
$$

- If one of the parameters ( $m$ or $k$ ) is odd and the other is even:

$$
1-\frac{6 G_{4}(m, k)}{\psi(m)}
$$

Computed values of this probability are listed in Table 4. Going deeper on them, the weight of the required difference of votes $k$ to guarantee a probability of transitive strict preferences equal to 1 with respect to the total number of individuals increases as the number of individuals does. To illustrate, in the case of $m=3$, the required $k=1$ represents around a $33.33 \%$ of the value of $m$ whereas in the case of $m=100,000$ the required $k$ represents a $99.998 \%$ of the value of $m$. In fact, the required differences are very large for all the considered cases with the exception of $m=3$. Even for $m=4$, the difference signifies a $50 \%$ of the value of $m$. For not too demanding differences, the probabilities increase in the cases of $m=4, m=5$ and $m=10$; this is not the case for $m=100, m=1,000$ and $m=100,000$ where asking reasonable differences of votes decreases the probability of transitive strict preferences.

Moreover, as in the case of the probabilities stated in Table 2, small variations in the magnitude of the differences of votes do not change, at least in a significant way, the probabilities. To illustrate, look at the probabilities of $m=100,000$ with differences $k$ equal to $0,1,2$ and 3 .

When large electorates are considered, the following corollary holds.

Corollary 2 For large electorates $(m \rightarrow \infty)$, the probability of transitive strict preference is as follows:

1. If $\alpha<1 / 3$

$$
1-\left(\frac{1}{16}+\frac{15}{16} \alpha-\frac{75}{8} \alpha^{2}+\frac{255}{8} \alpha^{3}-\frac{795}{16} \alpha^{4}+\frac{483}{16} \alpha^{5}\right) .
$$


Table 4 Probability of transitive $P_{k}^{L}$.

\begin{tabular}{|c|c|c|c|c|c|c|c|}
\hline $\begin{array}{r}m \rightarrow \\
k \downarrow\end{array}$ & 3 & 4 & 5 & 10 & 100 & 1,000 & 100,000 \\
\hline 0 & 0.9643 & 0.9524 & 0.9524 & 0.9161 & 0.9293 & 0.9366 & 0.9375 \\
\hline 1 & 1 & 0.9524 & 0.9762 & 0.9161 & 0.9293 & 0.9366 & 0.9375 \\
\hline 2 & & 1 & 0.9762 & 0.9580 & 0.9175 & 0.9348 & 0.9375 \\
\hline 3 & & & 1 & 0.9580 & 0.9175 & 0.9348 & 0.9375 \\
\hline 8 & & & & 1 & 0.9088 & 0.9298 & 0.9374 \\
\hline 98 & & & & & 1 & 0.9101 & 0.9366 \\
\hline 998 & & & & & & 1 & 0.9290 \\
\hline 99,998 & & & & & & & 1 \\
\hline
\end{tabular}

2. If $1 / 3 \leq \alpha<1$

$$
1-\frac{3}{16}(1-\alpha)^{5}
$$

Table 5 Probability of transitive $P_{k}^{L}$ for large electorates.

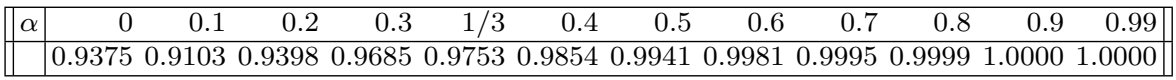

Table 5 sets forth some numerical values of the probability of transitive strict preferences for large electorates, as a function of the relative threshold $\alpha$. These results illustrate that the probabilities increase as $\alpha$ rises with the exception of the case of $\alpha=0.1$ in which the probability decreases with respect to the one for $\alpha=0$. More precisely, the probability function of transitive strict preferences for large electorates reaches its minimum 0.9080 at $\alpha=0.0737$. In addition, notice that the variations of the probabilities are relatively small as $\alpha$ increases.

3.3 Probabilities of transitive weak preferences under majorities based on difference of votes with linear orderings

To derive the probability of transitive weak preferences under $M_{k}^{L}$ majorities, we need to consider, in addition with the cases analyzed in Proposition 2, the cases from 14 to 19 in Table 1 . With that, we calculate the probability of nontransitive weak preferences and therefore, the probability of transitive weak preferences is determined as 1 minus the probability of non-transitive weak preferences.

By symmetry arguments, the weak preferences represented in cases from 14 to 19 are similar and therefore the number of integer solutions of the six systems of inequalities corresponding to such cases is the same. Using again the Barvinok's algorithm, two validity domains can be considered. If $k \leq(m-2) / 3$, 
the number of voting situations inside each system is given by $H_{1}(m, k)$ if both $m$ and $k$ are odd (or even), and by $H_{2}(m, k)$ if one of the parameters ( $m$ or $k)$ is odd and the other one is even such that:

$$
\begin{aligned}
H_{1}(m, k)= & -\frac{1}{240}\left(( k + 1 ) \left(17 k^{4}-30 m k^{3}-22 k^{3}+20 m^{2} k^{2}-\right.\right. \\
28 k^{2}+30 m k^{2}+50 m k+48 k-5 m^{3} k-5 m^{2} k- & \\
5 & \left.\left.m^{3}-30 m^{2}-40 m\right)\right) . \\
H_{2}(m, k)= & -\frac{1}{240} k\left(17 k^{4}-30 m k^{3}-90 k^{3}+20 m^{2} k^{2}+\right. \\
& 120 m k^{2}+140 k^{2}-5 m^{3} k-45 m^{2} k-100 m k- \\
& \left.30 k-37-5 m^{2}-30 m\right) .
\end{aligned}
$$

For the second validity domain, if $(m-1) / 3 \leq k \leq m-1$, this number is given by $H_{3}(m, k)$ if both $m$ and $k$ are odd (or even) and by $H_{4}(m, k)$ if one of the parameters $(m$ or $k$ ) is odd and the other one is even such that:

$$
\begin{aligned}
H_{3}(m, k)= & \frac{1}{3840}((m-k+2)(m-k)(m-k+4) \\
& \left.\left(29 k^{2}+12 m k+94 k+72-m^{2}+6 m\right)\right) . \\
H_{4}(m, k)= & \frac{1}{3840}((m-k+1)(m-k+5)(m-k+3) \\
& \left.\left(29 k^{2}+12 m k+36 k-6 m+7-m^{2}\right)\right) .
\end{aligned}
$$

Taking into consideration the intersections between the different validity domains and using the results in Propositions 1 and 2, the probability of transitive weak preferences is as follows.

Proposition 3 Consider a three-candidate election with $m$ voters under $M_{k}^{L}$ majority rule where each individual vote consists of a linear preference ordering on the candidates. Assuming that all voting situations are equally likely (IAC), the probability of transitive weak preferences is as follows:

1. If $k \leq(m-3) / 3$

- If both $m$ and $k$ are odd (or even):

$$
1-\frac{2 F_{1}(m, k)+6 G_{1}(m, k)+6 H_{1}(m, k)}{\psi(m)} .
$$

- If one of the parameters ( $m$ or $k$ ) is odd and the other one is even:

$$
1-\frac{2 F_{2}(m, k)+6 G_{2}(m, k)+6 H_{2}(m, k)}{\psi(m)} .
$$


2. If $(m-1) / 3 \leq k \leq m-2$

- If both $m$ and $k$ are odd (or even):

$$
1-\frac{6 G_{3}(m, k)+6 H_{3}(m, k)}{\psi(m)} .
$$

- If one of the parameters ( $m$ or $k$ ) is odd and the other one is even:

$$
1-\frac{6 G_{4}(m, k)+6 H_{4}(m, k)}{\psi(m)} .
$$

3. If $k=(m-2) / 3$

- Either both $m$ and $k$ are odd or both are even:

$$
1-\frac{6 H_{1}(m, k)+6 G_{3}(m, k)}{\psi(m)} .
$$

4. If $k=m-1$

- One of the parameters ( $m$ or $k$ ) is odd and the other one is even:

$$
1-\frac{6 H_{4}(m, k)}{\psi(m)} \text {. }
$$

Analyzing the probabilities of transitive weak preferences displayed in Table $6, M_{0}^{L}$ majority provides the highest values for the probability of having transitive weak preferences for almost all the considered values of $m$. In fact, any difference of votes can be asked to guarantee a probability value of 1 . Only in the cases of $m=1,000$ and $m=100,000$ the probability arrives to the value of 1.0000 , i.e. the probability approximates to the value of 1 without reaching it. Even so, in both cases the required difference of votes is extremely large. Specifically, it represents a $99.9 \%$ of the value of $m$ in the case of $m=1,000$ and a $99.999 \%$ in the case of $m=100,000$.

Table 6 Probability of transitive $R_{k}^{L}$.

\begin{tabular}{||r|rrrrrrr}
\hline $\begin{array}{r}m \rightarrow \\
k \downarrow\end{array}$ & 3 & 4 & 5 & 10 & 100 & 1,000 & 100,000 \\
\hline 0 & 0.9643 & 0.7619 & 0.9524 & 0.8462 & 0.9280 & 0.9366 & 0.9375 \\
2 & 0.6786 & 0.7143 & 0.6667 & 0.6703 & 0.9062 & 0.9346 & 0.9375 \\
3 & & 0.7143 & 0.7619 & 0.6703 & 0.9062 & 0.9346 & 0.9375 \\
4 & & & 0.7619 & 0.6503 & 0.8818 & 0.9327 & 0.9375 \\
9 & & & & 0.9101 & 0.8292 & 0.9287 & 0.9374 \\
99 & & & & & 0.9997 & 0.8136 & 0.9366 \\
999 & & & & & & 1.0000 & 0.9276 \\
99,999 & & & & & & & 1.0000
\end{tabular}

Finally, as in the previous cases stated in Tables 2 and 4, the probabilities do not significantly change with small variations of the magnitude of the difference in votes. On this, see for instance the cases of $k$ equal 2 and 3 for $m$ equal 4,10,100,1,000 and 100,000.

When large electorates are considered, the following corollary holds. 
Corollary 3 For large electorates $(m \rightarrow \infty)$, the probability of transitive weak preference is as follows:

1. If $\alpha<1 / 3$

$$
1-\left(\frac{1}{16}+\frac{15}{16} \alpha+\frac{45}{8} \alpha^{2}-\frac{225}{8} \alpha^{3}+\frac{645}{16} \alpha^{4}-\frac{333}{16} \alpha^{5}\right) .
$$

2. If $1 / 3 \leq \alpha<1$

$$
1-\frac{15}{8} \alpha(1-\alpha)^{3}(1+3 \alpha) .^{5}
$$

Table 7 Probability of transitive $R_{k}^{L}$ for large electorates.

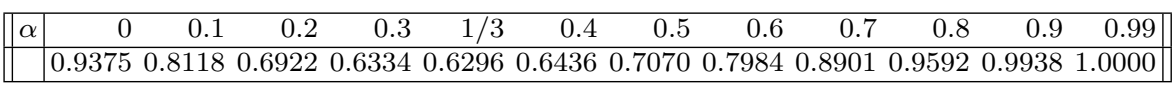

Table 7 presents some numerical values of the probability of transitive weak preferences for large electorates, as a function of the relative threshold $\alpha$. These results illustrate that the probabilities rapidly decrease in $\alpha$ and reach the minimum 0.6296 at $\alpha=1 / 3$. Instead, from $\alpha>1 / 3$, the probabilities increase as $\alpha$ rises.

\section{Probabilities of consistent collective decisions under majorities based on difference of votes with weak orderings}

Given that voters could be indifferent between the alternatives, we have to take into account the six linear preference orderings in (2), the six possible orderings that collect the partial indifference and the one that represents the complete indifference among three alternatives. Therefore,

$$
\begin{array}{lll}
x_{1} x_{2} x_{3}\left(m_{1}\right) & x_{1} x_{3} x_{2}\left(m_{2}\right) & x_{2} x_{1} x_{3}\left(m_{3}\right) \\
x_{2} x_{3} x_{1}\left(m_{4}\right) & x_{3} x_{1} x_{2}\left(m_{5}\right) & x_{3} x_{2} x_{1}\left(m_{6}\right) \\
\left\{x_{1} x_{2}\right\} x_{3}\left(m_{7}\right) & \left\{x_{1} x_{3}\right\} x_{2}\left(m_{8}\right) & \left\{x_{2} x_{3}\right\} x_{1}\left(m_{9}\right) \\
x_{1}\left\{x_{2} x_{3}\right\}\left(m_{10}\right) & x_{2}\left\{x_{1} x_{3}\right\}\left(m_{11}\right) & x_{3}\left\{x_{1} x_{2}\right\}\left(m_{12}\right) \\
\left\{x_{1} x_{2} x_{3}\right\}\left(m_{13}\right) & &
\end{array}
$$

where $m_{i}$ represents the number of voters with the associated preference ordering and $\left\{x_{i} x_{j}\right\}$ stands for the indifference between the alternatives $x_{i}$ and $x_{j}$. As the IAC condition is assumed, all possible voting situations $\boldsymbol{m}=$ $\left(m_{1}, m_{2}, \cdots, m_{13}\right)$ are equally liked to be observed. For $m$ individuals and

\footnotetext{
5 Notice that in this case, the four original validity domains from Proposition 3 reduce to two, given that, for the three last ones, the probabilities are the same when considering large electorates.
} 
3 alternatives, if the indifference between alternatives is allowed, the total number of voting situations $\boldsymbol{m}$ is given by the expression:

$$
\Psi(m)=\frac{(m+1)(m+2) \cdots(m+12)}{12 !} .
$$

Using the same approach applied in Section 3, the probability of consistent outcomes is calculated by means of the computation of the probability of inconsistent outcomes. As there, such probabilities are given by Ehrhart polynomials that provide the number of integer points inside the systems of inequalities that characterize each of the analyzed inconsistent outcomes.

In the framework of the preferences represented in (4), the complexity of the conditions makes impossible the derivation of a general mathematical representation as the one provided in Section 3 even for the case of large electorates. This is because for each considered consistency condition, the number of validity domains and the length of the polynomials are greater than in the cases of Section 3.

Fortunately, when the number of individuals $m$ and the threshold $k$ are fixed, the probabilities can be calculated for the given number of voting situations $\boldsymbol{m}$.

4.1 Probabilities of triple-acyclic strict preferences under majorities based on difference of votes with weak orderings

In Table 8, the probabilities of triple-acyclic strict preferences under $M_{k}$ majorities are displayed.

It must be emphasized that, in the case of $k=0$, the probability $0.9956=$ $1-0.0044$, as well as $0.9929=1-0.0071$ and $0.9571=1-0.042^{6}$, are in accordance with the results previously obtained by Lepelley and Martin (2001) when the number of voters equals 3 and 5 and tends to infinity, respectively.

Table 8 Probability of triple-acyclic $P_{k}$.

\begin{tabular}{||r|rrrrrrr}
\hline $\begin{array}{r}m \rightarrow \\
k \downarrow\end{array}$ & 3 & 4 & 5 & 10 & 100 & 1,000 & 100,000 \\
\hline 0 & 0.9956 & 0.9989 & 0.9929 & 0.9873 & 0.9627 & 0.9577 & 0.9571 \\
1 & 1 & 1 & 1 & 0.9992 & 0.9720 & 0.9589 & 0.9572 \\
2 & & & & 1.0000 & 0.9794 & 0.9601 & 0.9572 \\
3 & & & & 1 & 0.9851 & 0.9613 & 0.9572 \\
33 & & & & & 1 & 0.9852 & 0.9576 \\
333 & & & & & & 1 & 0.9611 \\
33,333 & & & & & & & \\
\hline
\end{tabular}

6 Notice that, in Lepelley and Martin (2001), the probability 0.042 is obtained as an estimate value through computer simulations. 
Furthermore, the following facts can be pointed out from these results. The probabilities of having triple-acyclic strict preferences when no difference of votes is required, i.e. when $M_{0}$ majority is applied, reach very high values. Specifically, they are located between 0.9571 and 0.9989 . For $m=3, m=4$ and $m=5$, the needed difference of votes to achieve a probability value of 1 equals 1. Therefore, it represents a one third of the value of $m$ in the case of $m=3$, a $25 \%$ in the case of $m=4$ and a $20 \%$ in the case of $m=5$. For the remaining considered values, the weight of the required differences represent around one third of the value of $m$ which means that we can guarantee with a probability of 1 the triple-acyclicity of strict preferences under $M_{k}$ majorities for reasonable values of the difference of votes.

4.2 Probabilities of transitive strict preferences under majorities based on difference of votes with weak orderings

In Table 9 , the probabilities of transitive strict preferences under $M_{k}$ majorities are presented.

Table 9 Probability of transitive $P_{k}$.

\begin{tabular}{|c|c|c|c|c|c|c|c|}
\hline $\begin{array}{r}m \rightarrow \\
k \downarrow\end{array}$ & 3 & 4 & 5 & 10 & 100 & 1,000 & 100,000 \\
\hline 0 & 0.9692 & 0.9626 & 0.9531 & 0.9422 & 0.9519 & 0.9565 & 0.9571 \\
\hline 1 & 1 & 0.9901 & 0.9884 & 0.9632 & 0.9443 & 0.9554 & 0.9571 \\
\hline 2 & & 1 & 0.9971 & 0.9851 & 0.9399 & 0.9542 & 0.9571 \\
\hline 3 & & & 1 & 0.9942 & 0.9383 & 0.9531 & 0.9571 \\
\hline 8 & & & & 1 & 0.9516 & 0.9484 & 0.9570 \\
\hline 98 & & & & & 1 & 0.9597 & 0.9560 \\
\hline 998 & & & & & & 1 & 0.9471 \\
\hline 99,998 & & & & & & & 1 \\
\hline
\end{tabular}

It is remarkable that to reach a probability of transitive strict preferences equal to 1 , the weight of the required difference of votes $k$ with respect to the total number of individuals increases as the number of individuals does. For instance, in the case of $m=4$, it represents a $50 \%$ of the value of $m$ whereas in the case of $m=1,000$ it does a $99.8 \%$ of the value of $m$.

Moreover, the required differences are too demanding for all the cases with the exception of the case of $m=3$ where it represents one third of the value of $m$.

4.3 Probabilities of transitive weak preferences under majorities based on difference of votes with weak orderings

Probabilities of transitive weak preferences under $M_{k}$ majorities defined for weak orderings are displayed in Table 10. 
Table 10 Probability of transitive $R_{k}$.

\begin{tabular}{||r|rrrrrrr}
\hline $\begin{array}{r}m \rightarrow \\
k \downarrow\end{array}$ & 3 & 4 & 5 & 10 & 100 & 1,000 & 100,000 \\
\hline 0 & 0.9801 & 0.8538 & 0.8920 & 0.9072 & 0.9511 & 0.9565 & 0.9571 \\
2 & 0.7890 & 0.7429 & 0.7014 & 0.6980 & 0.9218 & 0.9540 & 0.9571 \\
3 & & 0.8648 & 0.8167 & 0.6814 & 0.9049 & 0.9527 & 0.9571 \\
4 & & & 0.9176 & 0.7234 & 0.8871 & 0.9514 & 0.9571 \\
9 & & & & 0.9919 & 0.7954 & 0.9444 & 0.9570 \\
99 & & & & & 1.0000 & 0.7782 & 0.9559 \\
999 & & & & & & 1.0000 & 0.9436 \\
99,999 & & & & & & & 1.0000
\end{tabular}

The probability value of 1 is almost achieved for the values of $m$ equal to $100,1,000$ and 100,000 . In these cases, the required differences in votes $k$ are so high that signify a $98 \%$ of the value of $m$ in the case of $m=100$, a $99.8 \%$ in the case of $m=1,000$ and a $99.998 \%$ in the case of $m=100,000$.

\section{Conclusion}

In this paper, we have computed the theoretical probabilities of consistent preferences under majorities based on difference of votes defined for both individual linear orderings and weak orderings. This paper contains two new contributions. First, we have found the needed thresholds which guarantee that the probability of consistent outcomes is close to 1 . Second, we have set forth the impact of weak orderings and linear orderings on the probability of consistent preferences. In the cases of transitive and triple-acyclic strict preferences, we have shown that probabilities are higher considering weak than linear orderings (see Tables 2, 4, 8 and 9). In the case of transitive weak preferences, the same is true when $k$ equals 1 and 2 and, with the exception of the case in which $m=4$, also when $k=0$.

Since we have studied the particular case of Impartial and Anonymous Culture (IAC), the extension of these results to other probabilistic hypothesis as Impartial Culture (IC) remains open. Moreover, the extension of this study to other majorities is of interest. More precisely, a similar analysis can be achieved for the case of Majorities based on difference of support (GarcíaLapresta and Llamazares (2010)). These majorities allow individuals to show their intensities of preferences on pairs of alternatives by means of reciprocal preferences through values in the unit interval. Under them, an alternative $x_{1}$ defeats another one $x_{2}$ if the sum of the intensities for $x_{1}$ exceeds the sum of intensities for $x_{2}$ by a threshold fixed before the election process. Theoretical-and quite negative-results about the consistency of the collective preferences under these majorities have been developed in Llamazares et al. (2013) and Llamazares and Pérez-Asurmendi (2015). Hence, the study of the likelihood of consistent outcomes under these majorities seems to be relevant. 
Acknowledgements The authors are grateful to Ahmad Fliti, José Luis García-Lapresta, Bonifacio Llamazares, Ana Pérez Espartero and Rachid Seghir for their valuable suggestions and comments. This work is partially supported by the Spanish Ministry of Economy and Competitiveness (Projects ECO2012-32178 and ECO2012-34202).

\section{References}

Balasko, Y., \& Crès, H. (1997). The probability of Condorcet cycles and super majority rules. J. Econ. Theory, 75, 237-270.

Cervone, D. P., Gehrlein, W. V., \& Zwicker, W. S. (2005). Which scoring rule maximizes Condorcet efficiency under IAC? Theor. Decis., 58, 145-185.

Condorcet, M. d. (1785). Essai sur l'Application de l'Analyse à la Probabilité des Décisions Rendues à la Pluralité des Voix. Paris: Imprimerie Royale.

Ferejohn, J. A., \& Grether, D. M. (1974). On a class of rational social decisions procedures. J. Econ. Theory, 8, 471-482.

Fishburn, P. C., \& Gehrlein, W. V. (1980). The Paradox of Voting: Effects of individual indifference and intransitivity. J. Public Econ., 14, 83-94.

García-Lapresta, J. L., \& Llamazares, B. (2001). Majority decisions based on difference of votes. J. Math. Econ., 35, 463-481.

García-Lapresta, J. L., \& Llamazares, B. (2010). Preference intensities and majority decisions based on difference of support between alternatives. Group Decis. Negot., 19, $527-542$.

Gehrlein, W. V. (1983). Condorcet's Paradox. Theor. Decis., 15, 161-197.

Gehrlein, W. V. (1997). Condorcet Paradox and the Condorcet efficiency of voting rules. Math. Jpn., 40, 173-199.

Gehrlein, W. V. (2009). Condorcet's Paradox with three candidates. In S. J. Brams, W. V. Gehrlein, \& F. S. Roberts (Eds.), The Mathematics of Preference, Choice and Order, (pp. 183-196). Springer Berlin Heidelberg.

Gehrlein, W. V., \& Fishburn, P. C. (1976). Condorcet's Paradox and anonymous preference profiles. Public Choice, 26, 1-18.

Gehrlein, W. V., \& Fishburn, P. C. (1980). Robustness of positional scoring over subsets of alternatives. Appl. Math. Optim., 6, 241-255.

Gehrlein, W. V., \& Fishburn, P. C. (1981). Constant scoring rules for choosing one among many alternatives. Qual. Quant., 15, 203-210.

Gehrlein, W. V., \& Fishburn, P. C. (1983). Scoring rule sensitivity to weight selection. Public Choice, 40, 249-261.

Houy, N. (2007). Some further characterizations for the forgotten voting rules. Math. Soc. Sci., 53, 111-121.

Lepelley, D., Louichi, A., \& Smaoui, H. (2008). On Ehrhart polynomials and probability calculations in voting theory. Soc. Choice Welfare, 30, 363-383.

Lepelley, D., \& Martin, M. (2001). Condorcet's Paradox for weak preference orderings. Eur. J. Polit. Econ., 17, 163-177.

Llamazares, B. (2006). The forgotten decision rules: Majority rules based on difference of votes. Math. Soc. Sci., 51, 311-326.

Llamazares, B., \& Pérez-Asurmendi, P. (2015). Triple-acyclicity in majorities based on difference in support. Inform. Sci., 299, 209-220

Llamazares, B., Pérez-Asurmendi, P., \& García-Lapresta, J. L. (2013). Collective transitivity in majorities based on difference in support. Fuzzy Sets Syst., 216, 3-15.

Tovey, C. A. (1997). Probabilities of preferences and cycles with super majority rules. J. Econ. Theory, 75, 271-279.

Verdoolaege, S., Seghir, R., Beyls, K. et al. (2004). Analytical computation of Ehrhart polynomials: Enabling more compiler analysis and optimizations. In Proceedings of International Conference on Compilers, Architecture, and Synthesis for Embedded Systems, (pp. 248-258). Washington D.C., USA.

Wilson, M. C., \& Pritchard, G. (2007). Probability calculations under the IAC hypothesis. Math. Soc. Sci., 54, 244-256. 